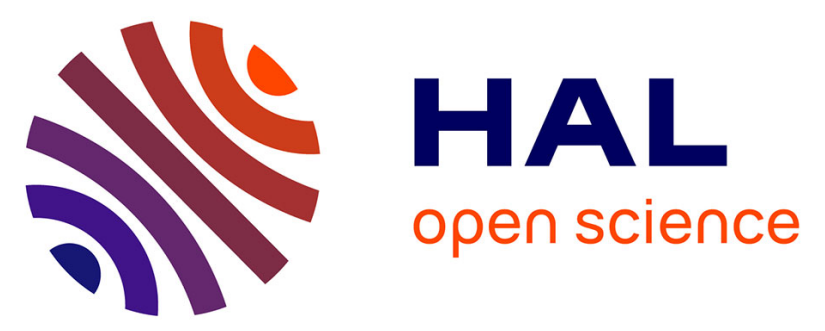

\title{
Bistatic MIMO System with Uniform Circular Arc Arrays for Single near Field Target Localization
}

Jinze Du, Bin Wu, Yi Yang, Chao Yuan, Parth Raj Singh, Amit Karel, Lian Li

\section{To cite this version:}

Jinze Du, Bin Wu, Yi Yang, Chao Yuan, Parth Raj Singh, et al.. Bistatic MIMO System with Uniform Circular Arc Arrays for Single near Field Target Localization. 10th International Conference on Communication Software and Networks (ICCSN 2018), Jul 2018, Chengdu, China. pp.357-360, 10.1109/ICCSN.2018.8488303 . lirmm-03127792

\section{HAL Id: lirmm-03127792 \\ https://hal-lirmm.ccsd.cnrs.fr/lirmm-03127792}

Submitted on 1 Apr 2021

HAL is a multi-disciplinary open access archive for the deposit and dissemination of scientific research documents, whether they are published or not. The documents may come from teaching and research institutions in France or abroad, or from public or private research centers.
L'archive ouverte pluridisciplinaire HAL, est destinée au dépôt et à la diffusion de documents scientifiques de niveau recherche, publiés ou non, émanant des établissements d'enseignement et de recherche français ou étrangers, des laboratoires publics ou privés. 


\title{
Bistatic MIMO System with Uniform Circular Arc Arrays for Single near Field Target Localization
}

\author{
Jinze Du \\ School of Mathematics and Statistics \\ Lanzhou University, China
}

\author{
Yi Yang \\ ${ }^{1}$ School of Information Science and Engineering, \\ Lanzhou University \\ ${ }^{2}$ Silk Road Economic Belt Research Center of Lanzhou \\ University, China
}

\author{
Parth Raj Singh \\ IETR \\ Polytech Nantes
}

France

\author{
$\mathrm{Bin} \mathrm{Wu}^{*}$ \\ Communication and Network Center \\ Lanzhou University, China \\ e-mail:wbin@1zu.edu.cn \\ Chao Yuan \\ IMPCAS \\ Institute of Modern Physics \\ Chinese Academy of Science, China
}

Amit Karel

LIRMM

University of Montpellier

France

Lian Li

School of Information Science and Engineering

Lanzhou University, China

\begin{abstract}
Near field sources cannot be located by using far field assumptions without compromising the precision. To solve this issue, many passive near field sources localization techniques have been proposed in the last few decades. There exists a lot of research work in which circular receiving array is used to locate a single near field source in 3D. In this paper, this domain is extended to active localization by proposing a low complexity closed-form method based on least squares estimation to locate a near field target using a bistatic MIMO system with uniform circular arc transmitting and receiving arrays. The simulation results indicate that the proposed method is capable to estimate the spherical coordinates of the target and also shows acceptable performance like the most of the existing near field source localization methods.
\end{abstract}

Keywords-near field target localization; uniform circular arc array; MIMO system

\section{INTRODUCTION}

A source in the space surrounding an antenna or an array falls under near or far field region based on its distance with respect to the largest dimension of the antenna or the array [1]-[3]. We can find enormous research work dealing with far field sources localization because it plays important role in the applications like radar, sonar, and wireless communication. However, near field sources localization is equally important. It has applications in medicine, robotics, smart devices, etc. Near field sources can not be located by using far field assumptions without compromising the precision. Therefore, many passive near field sources localization techniques have been proposed in the last few decades. Among which, there is a unique interest in single near field source localization techniques using uniform circular array (UCA) because of its attractive geometry and capability to localize source in $3 \mathrm{D}$ as compared to a unifrom linear array (ULA) [4]-[7].

In this paper, we extend this scenario to active localization by using a bistatic MIMO system with uniform circular arc transmitting and receiving arrays. All the antennas of both arrays are considered to lie on a big circle, forming two separate arcs on the circle for each array. This configuration simply replaces the circular array, therefore, this can be used in the applications of circular array. Classically, a 3D MUSIC based approach can be used to obtain a good estimation of the range, elevation angle, and azimuthal angle of a near field target, however, it will be computationally very costly due to three non-separable variables. Therefore, we propose a least squares (LS) based closed-form approach.

In the following, we formulate the signal model of the received signal reflected by a target in the near field region of a bistaic MIMO system with uniform circular arc array in section 2. In section 3, we explain the proposed method. Followed by, simulation results and conclusion in section 4 and section 5 respectively.

\section{NOTATIONS}

The scalars are represented by normal lower or upper case alphabets. A bold lowercase alphabet represents a 
vector whereas a bold uppercase alphabet denotes a matrix. $\mathbb{Z}, \mathbb{R}$, and $\mathbb{C}$ are the sets of all integers, real, and complex numbers respectively. $[\cdot]^{T}$ and $[\cdot]^{*}$ respectively denote the transpose and conjugate of a matrix. $|\cdot|, \arg (\cdot)$, and $\angle(\cdot)$ represent the absolute value, argument, and principal value of a complex number. $\mathrm{E}\{\cdot\}$ gives the expected value. $j=\sqrt{-1}$.

\section{SIGNAL MODEL}

Consider a bistatic MIMO system with uniform circular arc transmitting and receiving arrays containing $2 M+1$ and $2 N+1$ omnidirectional antennas respectively. The inter element spacing in both arrays is $d$ as shown in Fig. 1. The center elements of the arrays are taken as their phase reference points which are located on the $\mathrm{y}$-axis at distance $b$ from the origin. As shown in Fig. 1, all the antennas are located on a circle of radius $b$ in xy-plane making two arcs corresponding to each array. Here, we have considered single target situation whose radial distance, $\pi / 2 \mathrm{rad}$ elevation angle, and azimuthal angle with respect to the origin are denoted by $r \in[0, \infty), \theta \in[0, \pi / 2 \mathrm{rad}]$, and $\varnothing \in(-\pi \mathrm{rad}, \pi \mathrm{rad}]$ respectively.

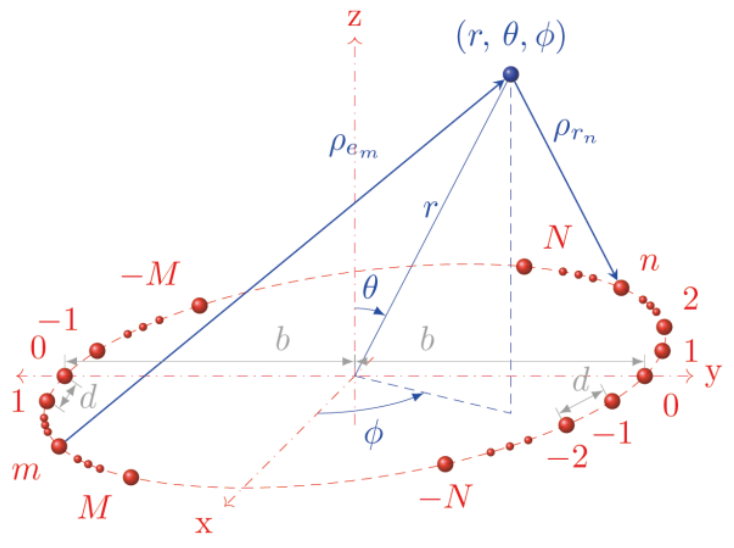

Figure 1. Uniform circular arc array

In case of a bistatic MIMO system, the matched filtered received signal for a single target can be written as [8]

$$
\mathbf{y}(t)=\left(\mathbf{a}_{\mathbf{e}} \otimes \mathbf{a}_{\mathbf{r}}\right) s(t)+\mathbf{w}(t)
$$

where $\mathbf{w}(t) \in \mathbb{C}^{(2 M+1)(2 N+1)}$ is the zero mean noise vector with variance $\sigma^{2}$ complex Gaussian entries, $s(t) \in \mathbb{C}$ is the complex reflection coefficient of the near field target varying with time $t$ according to Swerling case II [9] . $\mathbf{a}_{\mathbf{e}} \in \mathbb{C}^{(2 M+1)}$ and $\mathbf{a}_{\mathbf{r}} \in \mathbb{C}^{(2 N+1)}$ are respectively the directional vectors of departure and arrival which can be expressed a

$$
\mathbf{a}_{\mathbf{e}}=\left[\begin{array}{c}
e^{-j 2 \pi\left(\rho_{e_{-M}}-\rho_{e_{0}}\right) / \lambda} \\
\vdots \\
1 \\
\vdots \\
e^{-j 2 \pi\left(\rho_{e_{m}}-\rho_{e_{0}}\right) / \lambda} \\
\vdots \\
e^{-j 2 \pi\left(\rho_{e_{M}}-\rho_{e_{0}}\right) / \lambda}
\end{array}\right]
$$

and

$$
\mathbf{a}_{\mathbf{r}}=\left[\begin{array}{c}
e^{-j 2 \pi\left(\rho_{r_{-N}}-\rho_{r_{0}}\right) / \lambda} \\
\vdots \\
1 \\
\vdots \\
e^{-j 2 \pi\left(\rho_{r_{n}}-\rho_{r_{0}}\right) / \lambda} \\
\vdots \\
e^{-j 2 \pi\left(\rho_{r_{N}}-\rho_{r_{0}}\right) / \lambda}
\end{array}\right]
$$

where $\lambda$ is the wavelength of the carrier. $\rho_{e_{m}}$ is the distance traveled by the signal transmitted by the $m$ th transmitting antenna to reach the target. $\rho_{r_{n}}$ is the distance traveled by the reflected signal from the target to the $n$th receiving antenna. $\quad m \in\{-M, \cdots,-1,0,1, \cdots, M\}$ and $n \in$ $\{-N, \cdots,-1,0,1, \cdots, N\}$ are the relative indexes of the antennas with respect to their corresponding reference antennas. In terms of $(r, \theta, \phi), \rho_{e_{m}}$ and $\rho_{r_{n}}$ can be expressed as (4), (5)

$$
\rho_{e_{m}}=\sqrt{b^{2}+r^{2}-2 b r \sin (\theta) \sin (m \alpha+\phi)}
$$

and

$$
\rho_{r_{n}}=\sqrt{b^{2}+r^{2}+2 b r \sin (\theta) \sin (n \alpha+\phi)}
$$

where

$$
\alpha=\arccos \left(1-d^{2} /\left(2 b^{2}\right)\right)
$$

In case of a single receiving array, near field region is defined by Fresnel region which is bounded by the following upper $\left(F_{H}\right)$ and lower $\left(F_{L}\right)$ bounds (3),

$$
F_{H}=2 D^{2} / \lambda
$$

and

$$
F_{L}=0.62 \sqrt{D^{3} / \lambda}
$$

with $D$, the largest dimension of the array. On extending this concept to a bistatic MIMO system, we can have three such regions. Two corresponding to the individual arrays and one for the whole system. For the whole system, $D=2 b$. And, for the transmitting and receiving arrays, $D=b \sqrt{2(1-\cos (2 M \alpha))}$ and $D=b \sqrt{2(1-\cos (2 N \alpha))}$ respectively.

\section{PROPOSED METHOD}

The following subvectors can be written from $\mathbf{y}(t)$,

$$
\mathbf{y}_{\mathbf{e}}(t)=\mathbf{a}_{\mathbf{e}} s(t)+\mathbf{w}_{\mathbf{e}}(t) \in \mathbb{C}^{(2 M+1)}
$$

and

$$
\mathbf{y}_{\mathbf{r}}(t)=\mathbf{a}_{\mathbf{r}} s(t)+\mathbf{w}_{\mathbf{r}}(t) \in \mathbb{C}^{(2 N+1)}
$$

where $\mathbf{w}_{\mathbf{e}}(t)$ and $\mathbf{w}_{\mathbf{r}}(t)$ are the respective noise subvectors. Further, we can say 


$$
\begin{aligned}
g_{e_{m}}= & E\left\{\left[y_{\mathbf{e}}\right]_{m}(t)\left[y_{\mathbf{e}}\right]_{0}^{*}(t)\right\} \\
& =E\left\{s(t) s^{*}(t)\right\}\left[\mathbf{a}_{\mathbf{e}}\right]_{m}+E\left\{\left[w_{\mathbf{e}}\right]_{m}(t)\left[w_{\mathbf{e}}\right]_{0}^{*}(t)\right\}
\end{aligned}
$$

and

$$
\begin{aligned}
g_{r_{n}}= & E\left\{\left[y_{\mathbf{r}}\right]_{n}(t)\left[y_{r}\right]_{0}^{*}(t)\right\} \\
& =E\left\{s(t) s^{*}(t)\right\}\left[\mathbf{a}_{\mathbf{r}}\right]_{n}+E\left\{\left[w_{\mathbf{r}}\right]_{n}(t)\left[w_{\mathbf{r}}\right]_{0}^{*}(t)\right\}
\end{aligned}
$$

where $\left[\mathbf{a}_{\mathbf{e}}\right]_{m}$ denotes the component of $\mathbf{a}_{\mathbf{e}}$ corresponding to the $m$ th transmitting antenna. The components of the remaining vectors are denoted in the similar manner.

The argument of a complex number can only be calculated in its principal form. The arguments and principal values of $g_{e_{m}}$ and $g_{r_{n}}$ are related as, $\arg \left(g_{e_{m}}\right)=$ $\angle\left(g_{e_{m}}\right)+2 k \pi$ and $\arg \left(g_{r_{n}}\right)=\angle\left(g_{r_{n}}\right)+2 k \pi$, where $k \in \mathbb{Z}$ denotes the number of rotations around the origin. From (11) and (12), we can say, $\angle\left(\left[\mathbf{a}_{e}\right]_{m}\right)=\angle\left(g_{e_{m}}\right)$ and $\angle\left(\left[\mathbf{a}_{r}\right]_{n}\right)=\angle\left(g_{r_{n}}\right)$. However, for the proposed method, we need $\arg \left(\left[\mathbf{a}_{e}\right]_{m}\right)$ and $\arg \left(\left[\mathbf{a}_{r}\right]_{n}\right)$. For a single complex number, deducing its argument from its principal value is impossible. However, for a vector of complex numbers with unambiguous phase differences between the consecutive components, we can use unwrapping algorithm proposed in [10] to deduce the arguments. The unwrapping of the principal values of vector components often adds a phase shift to all the unwrapped phases. This phase shift can easily be removed by subtracting the unwrapped phase corresponding to the reference antenna from every unwrapped phase of that directional vector.

Let $\quad \beta_{e_{m}}=-\lambda \arg \left(\left[\mathbf{a}_{e}\right]_{m}\right) /(2 \pi)=\rho_{e_{m}}-\rho_{e_{0}} \quad$ and $\beta_{r_{n}}=-\lambda \arg \left(\left[\mathbf{a}_{r}\right]_{n}\right) /(2 \pi)=\rho_{r_{n}}-\rho_{r_{0}}$. Further, using (4) and (5) with some mathematical manipulations, we can transform these equations as

$$
\begin{aligned}
-\beta_{e_{m}}^{2} / 2= & b \sin (m \alpha) r \sin (\theta) \cos (\phi) \\
& +b(\cos (m \alpha)-1) r \sin (\theta) \sin (\phi) \\
& +\beta_{e_{m}} \sqrt{b^{2}+r^{2}-2 b r \sin (\theta) \sin (\phi)}
\end{aligned}
$$

and

$$
\begin{aligned}
-\beta_{r_{n}}^{2} / 2= & -b \sin (n \alpha) r \sin (\theta) \cos (\phi) \\
& -b(\cos (n \alpha)-1) r \sin (\theta) \sin (\phi) \\
& +\beta_{r_{n}} \sqrt{b^{2}+r^{2}+2 b r \sin (\theta) \sin (\phi)} .
\end{aligned}
$$

For all the values of $m$ and $n$, we can generate an overdetermined system of linear equations with four unknowns. Let

$$
\boldsymbol{\Gamma}=\left[\begin{array}{cccc}
b \sin (-M \alpha) & b \cos (-M \alpha)-b & \beta_{e_{-M}} & 0 \\
\vdots & \vdots & \vdots & \vdots \\
b \sin (M \alpha) & b \cos (M \alpha)-b & \beta_{e_{M}} & 0 \\
-b \sin (-N \alpha) & b-b \cos (-N \alpha) & 0 & \beta_{r_{-N}} \\
\vdots & \vdots & \vdots & \vdots \\
-b \sin (N \alpha) & b-b(\cos (N \alpha) & 0 & \beta_{r_{N}}
\end{array}\right]
$$

$$
\mathbf{x}=\left[\begin{array}{c}
r \sin (\theta) \cos (\phi) \\
r \sin (\theta) \sin (\phi) \\
\sqrt{b^{2}+r^{2}-2 b r \sin (\theta) \sin (\phi)} \\
\sqrt{b^{2}+r^{2}+2 b r \sin (\theta) \sin (\phi)}
\end{array}\right]
$$

and

$$
\Psi=\left[\begin{array}{c}
-\beta_{e_{-M}}^{2} / 2 \\
\vdots \\
-\beta_{e_{M}}^{2} / 2 \\
-\beta_{r_{-N}}^{2} / 2 \\
\vdots \\
-\beta_{r_{N}}^{2} / 2
\end{array}\right]
$$

Then, the system of linear equations can be written as

$$
\Gamma \mathbf{x}=\psi
$$

In (18), $\beta_{e_{m}}$ and $\beta_{r_{n}}$ appear on the either sides of the equations, therefore, total least squares (TLS) is a better approach here than the conventional least squares (LS) [11]. Let $\mathbf{v}=\left[v_{1}, v_{2}, v_{3}, v_{4}, v_{5}\right]^{T}$ be the right-singular-vector corresponding to the smallest singular value of $[\Gamma \mid \Psi] \in$ $\mathbb{R}^{2(M+N+1) \times 5}$. Then, the estimated radial range

$$
\hat{r}=\sqrt{\left(\left(v_{3} / v_{5}\right)^{2}+\left(v_{4} / v_{5}\right)^{2}-2 b^{2}\right) / 2}
$$

$\pi / 2$ - elevation angle

$$
\widehat{\theta}=\arcsin \left(\sqrt{\left(v_{1} / v_{5}\right)^{2}+\left(v_{2} / v_{5}\right)^{2}} / \hat{r}\right)
$$

and azimuthal angle

$$
\widehat{\phi}=\angle\left(-\left(v_{1} / v_{5}\right)-j\left(v_{2} / v_{5}\right)\right)
$$

\section{Simulation Results}

Consider the system shown in Fig. 1 with $7(M=3)$ transmitting and $9(N=4)$ receiving antennas. The inter element spacing, $d=\lambda / 2$. Through out the simulation, $\lambda$ is taken as the unit of length and the angles are expressed in degrees. Based on the considered radar parameters, the Fresnel bounds of the system and transmitting and receiving arrays are given in Table I.

\section{TABLE I. FRESNEL BOUNDS}

\begin{tabular}{ccc}
\hline & $\boldsymbol{F}_{\boldsymbol{H}}$ & $\boldsymbol{F}_{\boldsymbol{L}}$ \\
\hline $\begin{array}{c}\text { Transmitting } \\
\text { array }\end{array}$ & $14.931 \lambda$ & $\mathbf{2 . 8} \boldsymbol{\lambda}$ \\
$\begin{array}{c}\text { Receiving } \\
\text { array }\end{array}$ & $22.7346 \lambda$ & $\mathbf{3 . 8 3 8 3} \boldsymbol{\lambda}$ \\
\hline System & $\mathbf{3 2 \lambda}$ & $\mathbf{4 . 9 6} \boldsymbol{\lambda}$
\end{tabular}

To analyze the performance of the proposed method, we have compared the root mean square errors (RMSE) in the estimations of $\rho, \theta$, and $\phi$ with their Cramér-Rao lower bounds (CRLB) for different signal-to-niose-ratios (SNR) in Fig. 2. $L=10^{3}$ samples and $K=10^{3}$ Monte Carlo trails are used to compute RMSE. 


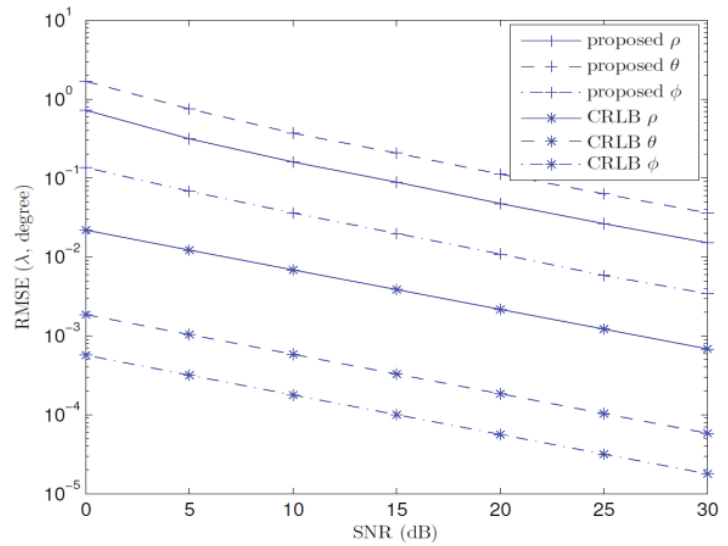

Figure 2. RMSE versus SNR; $d=\lambda / 2, K=10^{3}, L=10^{3}, M=3, N=4$, $r=5 \lambda, \theta=60^{\circ}$, and $\phi=70^{\circ}$.

From Fig. 2, we can say that the proposed method is capable to estimate the spherical coordinates of the target. However, the RMSE of target parameters are far from their respective CRLB. The primary reason of this poor performance is the partial exploitation of the available information.

\section{CONCLUSION}

A low complexity single target localization method using a passive radar system is proposed here. The arrays of the bistatic MIMO system are assumed to lie on a big imaginary circle to simplify the model which allows us to estimate the spherical coordinates of the target using closed from expressions. The proposed method can also show acceptable performance for $d=\lambda / 4$, like the most of the existing near field source localization methods.

\section{ACKNOWLEDGEMENT}

The authors would like to express their gratitude to all the subjects that participated in the experiments. This study is supported by Science and Technology Innovation Project of Foshan City, China (Grant No. 2015IT100095), the
Fundamental Research Funds for the Central Universities (Grant No. lzujbky-2016-br03), CERNET Innovation Project (Grant No. NGII20150603) and Science and Technology Planning Project of Guangdong Province, China (Grant No. 2016B010108002).

\section{REFERENCES}

[1] A. L. Swindlehurst and T. Kailath, "Passive direction-of-arrival and range estimation for near-field sources," in Fourth Annual ASSP Workshop on Spectrum Estimation and Modeling, Minneapolis, Minnesota, August 1988, pp.123-128.

[2] E. Grosicki, K. Abed-Meraim, and Y. Hua, "A weighted linear prediction method for near-field source localization," IEEE Trans. Signal Process, vol.53, no .10, pp.3651-3660, 2005.

[3] W. Zhi and M.-W. Chia, "Near-field source localization via symmetric subarrays," IEEE Signal Process Letters, vol. 14, no. 6, pp. 409-412, June 2007.

[4] T.-J. Jung and K. Lee, "Closed-form algorithm for 3-D single-source localization with uniform circular array," IEEE Antennas and Wireless Propagation Letters, vol.13, pp. 1096-1099, June 2014.

[5] Y. Wu, H. Wang, L. Huang, and H. C. So, "Fast algorithm for threedimensional single near-field source localization with uniform circular array," in IEEE CIE International Conference on Radar, vol. 1. Chengdu, China: IEEE, October 2011, pp.350-352.

[6] B. Eun-Hyon and L. Kyun-Kyung, "Closed-form 3-D localization for single source in uniform circular array with a center sensor," IEICE transactions on communications, vol. 92, no. 3, pp.1053-1056, 2009.

[7] Y. Wu and H. C. So, "Simple and accurate two-dimensional angle estimation for a single source with uniform circular array," IEEE Antennas and Wireless Propagation Letters, vol. 7, pp. 78-80, April 2008.

[8] H. Yan, J. Li, and G. Liao, "Multitarget identification and localization using bistatic MIMO radar systems," EURASIP Journal on Advances in Signal Processing, p. 48, 2008.

[9] P. Swerling, "Probability of detection for fluctuating targets," IRE Transactions on Information Theory, vol. 6, no .2, pp.269-308, April 1960.

[10] J. Tribolet, "A new phase unwrapping algorithm," IEEE Transactions on Acoustics Speech and Signal Processing, vol. 25, no .2, pp. 170177, April 1977.

[11] G. H. Golub and C. V. Loan, "Total least squares," in Smoothing Techniques for Curve Estimation. Springer, 1979, pp. 69-76 